\title{
Expression of HIF-1 $\alpha$ is a predictive marker of the efficacy of neoadjuvant chemotherapy for locally advanced cervical cancer
}

\author{
BIN YAN $^{1}$, QUAN-FU MA ${ }^{1}$, WEN-FU TAN ${ }^{1}$, HONG-NING CAI $^{1}$, YAN-LI LI ${ }^{1}$, ZHI-GANG ZHOU $^{1}$, XUAN DAI $^{1}$, \\ FA-XIA ZHU ${ }^{1}$, YU-JING XIONG ${ }^{1}$, MENG XU $^{1}$, YU-LIN GUO ${ }^{1}$, HAN GAO $^{1}$, JUN-BO HU $^{2}$ and XU-FENG WU ${ }^{1}$
}

Departments of ${ }^{1}$ Gynecologic Oncology and ${ }^{2}$ Pathology, Maternal and Child Health Hospital of Hubei Province, Huazhong University of Science and Technology, Wuhan, Hubei 430070, P.R. China

Received July 5, 2019; Accepted April 7, 2020

DOI: $10.3892 / \mathrm{ol} .2020 .11596$

\begin{abstract}
Platinum-based, arterial infusion chemotherapy as a neoadjuvant chemotherapy (NACT) followed by hysterectomy may be efficient for the treatment of locally advanced cervical cancer and improve prognosis. It is important to predict whether the NACT would be effective before it is launched. Hypoxia inducible factor- $1 \alpha$ (HIF-1 $\alpha$ ) is the master transcriptional regulator of the cellular response to altered oxygen concentration. HIF-1 $\alpha$ protein expression is elevated in numerous human malignancies, contributes to poor disease outcome, and has been reported to induce tumorigenesis and chemoresistance. In the present study, patients with International Federation of Gynecology and Obstetrics stage IIB-IIIB cervical cancer $(n=59)$ between 2008 and 2014 were assessed for HIF-1 $\alpha$ expression by immunohistochemistry. Tumor samples were obtained by biopsy before any treatment. A double-path chemotherapy regimen, paclitaxel (intravenous) plus cisplatin (intra-arterial injection into the uterine region), was used as NACT. The patients were then separated into two groups according to NACT response: One group comprised patients with NACT, for whom the response to treatment was efficient resulting in complete/partial remission of the tumor ( $\mathrm{CR}+\mathrm{PR}$ group; $\mathrm{n}=52$ ), the other group contained patients with NACT, for whom the result of the treatment was a stable/progressive disease (SD + PD group; $n=7$ ). HIF-1 $\alpha$ expression was tested in paraffin-embedded sections using immunohistochemistry.
\end{abstract}

Correspondence to: Professor Xu-Feng Wu, Department of Gynecologic Oncology, Maternal and Child Health Hospital of Hubei Province, Huazhong University of Science and Technology, 745 Wuluo Road, Wuhan, Hubei 430070, P.R. China

E-mail: zwuxufeng@163.com

Dr Jun-Bo Hu, Department of Pathology, Maternal and Child Health Hospital of Hubei Province, Huazhong University of Science and Technology, 745 Wuluo Road, Wuhan, Hubei 430070, P.R. China E-mail: 598747929@qq.com

Key words: hypoxia-inducible factor-1 $\alpha$, neoadjuvant chemotherapy, cervical cancer, predictive marker, prognosis
HIF-1 $\alpha$ expression was significantly higher in the SD + PD group compared with the $\mathrm{CR}+\mathrm{PR}$ group $(\mathrm{P}=0.029)$. The overall survival time was significantly longer in the $\mathrm{CR}+\mathrm{PR}$ group compared with the $\mathrm{SD}+\mathrm{PD}$ group $(\mathrm{P}<0.001)$. When the patients were divided into two groups based on HIF-1 $\alpha$ expression levels. Low (weighted score $\leq 4, n=39$ ) and high (weighted score $\geq 6, n=20$ ) expression level groups; the low HIF-1 $\alpha$ expression group was significantly more susceptible to NACT treatment $(\mathrm{P}=0.025)$. Cox hazard analysis revealed that a high level of HIF-1 $\alpha$ expression and lymph node metastases were significant independent predictors of poor overall survival $(\mathrm{P}=0.025, \mathrm{HR}=6.354 ; \mathrm{P}=0.020, \mathrm{HR}=6.909$, respectively). These results indicated that the expression of HIF-1 $\alpha$ may be able to predict the efficiency of NACT and may be considered an independent prognostic factor for stage IIB-IIIB cervical cancer.

\section{Introduction}

According to cervical cancer clinical guidelines, since 1999 the standard treatment for patients with locally advanced cervical cancer (LACC), defined as International Federation of Gynecology and Obstetrics (FIGO) stage $\geq \mathrm{IIB}$, is platinum-based concurrent chemoradiotherapy (CCRT) (1); however, the prognosis is still unsatisfactory. Several studies have demonstrated a $40-60 \%$ reduction in the relative risk of recurrence and a $30-50 \%$ reduction of the risk of death with CCRT (2-4).

Platinum-based neoadjuvant chemotherapy (NACT), followed by radical hysterectomy, has been reported to be effective in patients with LACC (5), with a prognosis equal to that of CCRT (6). Furthermore, a previous study reported that patients who had a good response to NACT had longer tumor-free survival and a lower recurrence rate than patients who had no response to NACT $(\mathrm{P}<0.001 ; \mathrm{P}=0.013)(7)$. However, chemoresistance to NACT is still a major challenge. For patients that do not respond to NACT, hysterectomy cannot be performed; consequently, the treatment strategy must be changed from surgery to radiation therapy, which results in a long period treatment delay, affecting prognosis (8-10). Therefore, it is important to identify prognostic factors in patients with LACC that predict whether NACT will be efficient before treatment (11-15). 
The development of hypoxia in solid tumors is associated with tumor progression, metastasis and recurrence following treatment (16). Hypoxia-inducible factor-1 (HIF-1) is the master transcriptional factor that regulates oxygen homeostasis (17). It comprises a constitutive $\beta$-subunit and an $\alpha$-subunit whose protein level depends on surrounding oxygen concentration. When oxygen is available, HIF-1 $\alpha$ is rapidly degraded (18). Under hypoxic conditions, HIF-1 $\alpha$ escapes degradation and rapidly dimerizes with HIF-1 $\beta$. The dimers subsequently translocate to the nucleus and regulate transcription of a series of hypoxia-dependent genes (19).

At the genetic level, HIF-1 $\alpha$ gene polymorphisms cause substantially higher transcriptional activity than the wild-type, and the C1772T polymorphism has been reported to be significantly related to response in patients undergoing NACT for LACC (20). At the protein level, HIF-1 $\alpha$ has been demonstrated to be upregulated in a wide range of solid tumors due to hypoxic conditions or aberrant activation of some oncogenes (21). Elevated HIF-1 $\alpha$ levels makes tumor cells more resistant to chemotherapy and increases the likelihood of metastasis and poor outcome (22). HIF-1 $\alpha$ expression has previously been shown to be associated with tumor stage and histology of cervical cancer (23). In addition, high expression of HIF-1 $\alpha$ resulted in worse 5-year survival rates than those patients with low HIF-1 $\alpha$ expression $(24,25)$. However, to date, to the best of our knowledge, there is no study available on the association between HIF-1 $\alpha$ protein expression and the chemoresistance of cervical cancer. To the best of our knowledge, the present study is the first to identify HIF- $1 \alpha$ protein expression as a biomarker of chemoresistance in patients with LACC.

The present study was designed to investigate whether the expression levels of HIF- $1 \alpha$ were associated with the chemoresistance of NACT for patients with FIGO stage IIB-IIIB LACC.

\section{Patients and methods}

Patients and samples. Between January 2008 and December 2014, >600 patients with cervical cancer were referred to the Gynecologic Oncology Department, Maternal and Child Health Hospital of Hubei Province (Wuhan, China). Patients received a standard evaluation, including physical and gynecological examination, colposcopy, biopsy, laboratory examinations and image examinations, including chest $\mathrm{X}$-ray, intravenous pyelography, and hepatic and pelvic ultrasonography. Exclusion criteria included the lack of informed consent, the lack of tumor samples, existing complicating disease or prior malignant disease, and patients who did not undergo NACT. Finally, 59 patients aged $<70$ years with complete data on age, clinical stage, grade, histology, size of tumor and main therapy, who had primary and previously untreated LACC (FIGO stages IIB-IIIB) were enrolled and analyzed retrospectively. The tumor samples were obtained by biopsy prior to any treatment. The tumor size was measured by the combination of pelvic examination and ultrasonography. Two senior oncological gynecologists participated in the evaluation. Written informed consent was obtained from all patients prior to the tumor biopsy. The Ethics Committee of Maternal and Child Healthcare
Hospital of the Hubei province approved the current study protocol.

NACT with transcatheter arterial chemoembolization (TACE) technology. NACT was administered using gelatin sponge particles (GSPs) combined with TACE (26) using the Seldinger technique (27), and a paclitaxel/cisplatin treatment regimen was applied. Briefly, a catheter (5-French diameter) was inserted into the left uterine artery region under the guidance of digital subtraction angiography to locate the tumor feeding vessels. Cisplatin $\left(75 \mathrm{mg} / \mathrm{m}^{2}\right)$ was divided into six doses, one dose was injected into the left uterine artery, one dose with 700-1,000 $\mu \mathrm{m}$ GSPs was injected into the peripheral uterine artery, then 2-3 mm GSPs were injected into the main uterine artery, and one dose was injected when the catheter came back to the anterior trunk of the iliac artery (not the superior gluteal artery). The same operation was done using the other three doses in the right uterine artery region, with adequate hydration prior to and following TACE to preserve renal function. After TACE, but on the same day, paclitaxel $\left(175 \mathrm{mg} / \mathrm{m}^{2}\right)$ was administered intravenously for $3 \mathrm{~h}$.

Treatment after NACT. NACT was administered for 1-3 cycles at 21-day intervals (between the start day of two cycles). One cycle of NACT was initially given. Only responders received the next cycle. A total of 2 weeks after each cycle, the clinical response to NACT and the operability was evaluated by magnetic resonance imaging and pelvic examination according to the World Health Organization (WHO) criteria, and defined as: Complete remission (CR), partial remission (PR), stable disease (SD) and progressive disease (PD) (28). NACT responders included patients with $\mathrm{CR}$ or PR, while non-NACT responders were patients with $\mathrm{SD}$ or PD. The cases were divided into two groups according to the efficiency of NACT: A complete/partial remission $(\mathrm{CR}+\mathrm{PR})$ group and a stable/progressive disease $(\mathrm{SD}+\mathrm{PD})$ group (Table I).

NACT responders could have surgery followed by radiation therapy; some patients chose to have radiation therapy directly due to age or financial reasons. Non-responders received radiation therapy after one cycle of NACT. According to their treatment after NACT, patients were divided into two groups: One group consisted of patients who had surgery and radiation therapy following NACT (NACT $+\mathrm{S}+\mathrm{R}$ group; $\mathrm{n}=40$ ); the other group consisted of patients where only radiation therapy was performed (NACT + R group; $n=19$ ) (Table I).

Blood counts, and liver and renal function exams were performed weekly, or more frequently if there was evidence of toxicity. Treatment was delayed if the white blood cell (WBC) count was $<3,000 / \mathrm{mm}^{3}$ or the platelet (PLT) count was $<100,000 / \mathrm{mm}^{3}$. The drug doses would be reduced by $20 \%$ if WBC count was $<1,000 / / \mathrm{mm}^{3}$ or PLT count was $<50,000 / \mathrm{mm}^{3}$ over a period of $>5$ days. Recombinant human granulocyte colony-stimulating factor was administered with persistent grade 3-4 myelotoxicity.

A total of 2 weeks after the last cycle, patients in the $\mathrm{NACT}+\mathrm{S}+\mathrm{R}$ group underwent type III radical hysterectomy with pelvic lymphadenectomy. For patients with squamous cancer and those $<40$ years old, one ovary was preserved and suspended outside the pelvis. The NACT $+\mathrm{S}+\mathrm{R}$ and 
Table I. Chemotherapeutic response according to clinicopathological parameters.

\begin{tabular}{|c|c|c|c|c|c|}
\hline \multirow[b]{2}{*}{ Variables } & \multirow[b]{2}{*}{ Total no. of patients } & \multicolumn{2}{|c|}{ Response to NACT } & \multirow[b]{2}{*}{ Response rate $(\%)$} & \multirow[b]{2}{*}{ P-value } \\
\hline & & $\mathrm{CR}+\mathrm{PR}$ & $\mathrm{SD}+\mathrm{PD}$ & & \\
\hline Number of patients & 59 & 52 & 7 & 88.14 & \\
\hline Age, years (range) & & $47(28-62)$ & $50(40-60)$ & & $0.284^{\mathrm{a}}$ \\
\hline \multicolumn{6}{|l|}{ Clinical stage } \\
\hline Stage IIB & 56 & 49 & 7 & 87.50 & $0.514^{\mathrm{b}}$ \\
\hline Stage IIIA & 0 & 0 & 0 & & \\
\hline Stage IIIB & 3 & 3 & 0 & 100 & \\
\hline \multicolumn{6}{|l|}{ Grade } \\
\hline G1 & 12 & 11 & 1 & 91.67 & $0.672^{\mathrm{b}}$ \\
\hline $\mathrm{G} 2 / \mathrm{G} 3$ & 47 & 41 & 6 & 87.23 & \\
\hline \multicolumn{6}{|l|}{ Histology } \\
\hline $\mathrm{SCC}$ & 53 & 49 & 4 & 92.45 & $0.017^{\mathrm{b}}$ \\
\hline A & 6 & 3 & 3 & 50 & \\
\hline AS & 0 & 0 & 0 & & \\
\hline Others & 0 & 0 & 0 & & \\
\hline \multicolumn{6}{|l|}{ Size of tumor } \\
\hline$<4 \mathrm{~cm}$ & 10 & 9 & 1 & 90 & $0.841^{\mathrm{b}}$ \\
\hline$\geq 4 \mathrm{~cm}$ & 49 & 43 & 6 & 87.76 & \\
\hline \multicolumn{6}{|l|}{ Main therapy } \\
\hline $\mathrm{NACT}+\mathrm{S}+\mathrm{R}$ & 40 & 38 & 2 & 95 & $0.053^{\mathrm{b}}$ \\
\hline $\mathrm{NACT}+\mathrm{R}$ & 19 & 14 & 5 & 73.68 & \\
\hline
\end{tabular}

${ }^{a}$ Independent two-sample t-test; ${ }^{\mathrm{b}} \chi^{2}$ test. $\mathrm{CR}+\mathrm{PR}$, complete remission + partial remission; $\mathrm{SD}+\mathrm{PD}$, stable disease + progressive disease; $\mathrm{NACT}+\mathrm{S}+\mathrm{R}$, neoadjuvant chemotherapy + surgery + radiotherapy; NACT + R, neoadjuvant chemotherapy + radiotherapy; FIGO, International Federation of Gynecology and Obstetrics; SCC, squamous cell carcinoma; A, adenocarcinoma; AS, adenosquamous carcinoma.

$\mathrm{NACT}+\mathrm{R}$ groups had radiotherapy after NACT + surgery or NACT directly.

Follow-up study. All patients were followed up periodically until May 2019. Overall survival was defined as the period of time from initial treatment until cervical cancer-related death. Surviving patients were censored on the date of the last follow-up.

Immunohistochemical analysis. The expression of HIF-1 $\alpha$ was detected in $4 \%$ formalin-fixed for $24 \mathrm{~h}$ at room temperature, paraffin-embedded sections (size, $4 \mu \mathrm{m}$ ) by immunohistochemical staining, as previously described (29).

Briefly, 4- $\mu \mathrm{m}$ paraffin-embedded sections were deparaffinized and immersed in $3 \%$ hydrogen peroxidase in methanol for $10 \mathrm{~min}$ at room temperature to block endogenous peroxidase activity. The antigen was retrieved by immersing the slides in $10 \mathrm{mM}$ citrate buffer ( $\mathrm{pH} \mathrm{6.0)}$ and heating at $110^{\circ} \mathrm{C}$ for $5 \mathrm{~min}$, followed by washing in PBS. The sections were then incubated with a monoclonal rabbit anti-human HIF-1 $\alpha$ antibody (clone EP1215Y; 1:200; cat. no. ab51608; Abcam) overnight at $4^{\circ} \mathrm{C}$. The samples were washed with PBS for $15 \mathrm{~min}$ and incubated with a HRP-conjugated anti-rabbit secondary antibody (1:200; cat. no. Sb 129; Servicebio) for $30 \mathrm{~min}$ at room temperature. 3,3'-diaminobenzidine was used as the chromogen for $5 \mathrm{~min}$ at room temperature. Finally, the sections were counterstained with Mayer's hematoxylin for $10 \mathrm{sec}$ at room temperature.

Two independent pathologists blinded to the clinical parameters used a light Olympus-IX71 microscope (magnification, x400; Olympus Corporation) to observe the images. HIF-1 $\alpha$ expression was semi-quantitatively analyzed based on the scoring method of Sinicrope et al (30). Briefly, the staining results were scored based on the following criteria: i) The percentage of positive staining was determined in five separate areas (magnification, $\mathrm{x} 400) ; 0(<5 \%), 1(5-25 \%)$, $2(25-50 \%), 3(50-75 \%)$ and $4(>75 \%)$; ii) staining intensity was scored as 0 (none), 1 (weak), 2 (moderate) and 3 (strong). The weighted score was calculated by multiplying the staining intensity score by the percentage of positive staining for each tissue specimen. The mean value of the weighted score was 5, so a weighted score of $0,1,2,3,4$ was defined as low HIF-1 $\alpha$ expression, and a weighted score of $6,8,9,12$ was defined as high HIF-1 $\alpha$ expression.

Statistical analysis. Data are presented as the mean \pm standard deviation. The Kaplan-Meier and log-rank tests were used for survival analysis and to determine the significance of differences in survival distribution. The weighted scores were compared using the Mann-Whitney U test. The independent two-sample t-test and a $\chi^{2}$ test were performed for intergroup 
Table II. Pathological findings from surgical specimens.

Response to NACT

\begin{tabular}{lcrr}
\cline { 2 - 3 } Positive rate & CR + PR & SD + PD & P-value $^{\mathrm{a}}$ \\
\hline Lymph node metastasis & $26.3 \%(10 / 38)$ & $100 \%(2 / 2)$ & 0.024 \\
Surgical margin & $10.5 \%(4 / 38)$ & $0 \%(0 / 2)$ & 0.629 \\
Vascular invasion & $2.6 \%(1 / 38)$ & $0 \%(0 / 2)$ & 0.816 \\
Depth of cervical invasion & $50 \%(19 / 38)$ & $0 \%(0 / 2)$ & 0.168 \\
\hline
\end{tabular}

${ }^{a} \chi^{2}$ test. $\mathrm{CR}+\mathrm{PR}$, complete remission + partial remission; SD + PD, stable disease + progressive disease.

comparisons. Univariate and multivariate Cox proportional hazard regression model was used to identify the potential independence predictors. SPSS software, version 21.0 (IBM, Corp.), was used for all the statistical analyses. $\mathrm{P}<0.05$ was considered to indicate a statistically significant difference.

\section{Results}

Patient characteristics and clinical response to NACT. As summarized in Table I, clinical chemotherapeutic response evaluation identified 52 patients as NACT responders (CR + PR group; 52/59; 88.14\%) and seven patients were identified as non-NACT responders (SD + PD group; $7 / 59 ; 11.86 \%)$. The association between response rate and clinicopathological parameters has been detailed in Table I. Squamous cell carcinoma exhibited a more favorable response than adenocarcinoma $(\mathrm{P}=0.017)$. Age, FIGO stage, grade, size of the tumor and main therapy did not exhibit significant differences in NACT response ( $\mathrm{P}>0.05$; Table I).

Pathological findings. The pathological findings were analyzed within the NACT $+\mathrm{S}+\mathrm{R}$ group using the specimens obtained after surgery. Significantly reduced pelvic lymph node metastasis was detected in the $\mathrm{CR}+\mathrm{PR}$ group compared with SD + PD group (26.3 vs. $100 \%$; $\mathrm{P}=0.024$; Table II). There were no significant differences in surgical margin rates, depth of cervical invasion rates and vascular invasion rates between the two groups $(\mathrm{P}>0.05$; Table II).

Expression of HIF-1 $\alpha$. HIF-1 $\alpha$ was expressed in the nuclei and cytoplasm of tumor cells (Fig. 1). The brown staining represents HIF-1 $\alpha$ expression, while blue staining represents the nuclei. The staining results were scored based on the following criteria: i) The percentage of positive staining was determined in five separate areas (magnification, $\mathrm{x} 400) ; 0(<5 \%), 1(5-25 \%)$, $2(25-50 \%), 3(50-75 \%)$ and 4 (>75\%); ii) staining intensity was scored as 0 (none), 1 (weak), 2 (moderate) and 3 (strong). The weighted score was calculated by multiplying the staining intensity score by the percentage of positive staining for each tissue specimen. The mean value of the weighted score was 5 , so a weighted score of $0,1,2,3,4$ was defined as low HIF-1 $\alpha$ expression, and a weighted score of $6,8,9,12$ was defined as high HIF-1 $\alpha$ expression. The mean weighted score for HIF-1 $\alpha$ expression was significantly lower in the CR + PR group compared with the SD + PD group (3.75 vs. 6.29;
$\mathrm{P}=0.029$; Fig. 2). In total, 39 of the 59 patients exhibited low expression levels of HIF-1 $\alpha$ (weighted scores, $0-4$ ), and 20 had high HIF-1 $\alpha$ expression (weighted score, 6-12). There were no significant differences in clinical characteristics observed between the two groups (Table III).

Association between the expression of HIF-1 $\alpha$ and the efficiency of NACT. Of the 39 patients with low HIF-1 $\alpha$ expression, 37 patients (94.87\%) were in the CR + PR group and two patients (5.13\%) were in the SD + PD group, whereas with regards to high HIF-1 $\alpha$ expression, $15 / 20$ patients (75\%) were in the $\mathrm{CR}+\mathrm{PR}$ group, and 5/20 (25\%) patients were in the SD + PD group. This indicated that patients with low HIF-1 $\alpha$ expression were significantly more responsive to NACT compared with patients with high HIF-1 $\alpha$ expression $(\mathrm{P}=0.025$; Table IV).

Survival. The overall survival time was significantly longer in the CR + PR group, compared with the SD + PD group $(\mathrm{P}<0.001 ;$ Fig. 3). The low HIF-1 $\alpha$ expression group exhibited significantly longer overall survival time compared with the high HIF-1 $\alpha$ expression group (P=0.017; Fig. 4).

Multivariate analysis in $N A C T+S+R$ group. A multivariate Cox proportional-hazard regression model was used to evaluate the relative strength and potential independence of HIF- $1 \alpha$ expression, NACT response and lymph node metastases using post-surgery specimens. Age, FIGO stage, size,-histology, surgical margin, depth of cervical invasion and vascular invasion had no significant impact on recurrent free survival (RFS) in univariate analysis (data not shown) and consequently were not included in multivariate analysis. High HIF-1 $\alpha$ expression levels and lymph node metastases were significant independent predictors of poor RFS, whereas response to NACT was not significant (Table V).

\section{Discussion}

Cervical cancer is a clinical and pathological heterogeneous malignancy, which requires different treatment strategies and has a variety of patient outcomes. For early-stage cervical cancer, surgery is accepted as the standard treatment. For the treatment of patients with LACC, CCRT is recommended as the standard treatment (31). However, limited access to radiation equipment, especially in developing countries, 

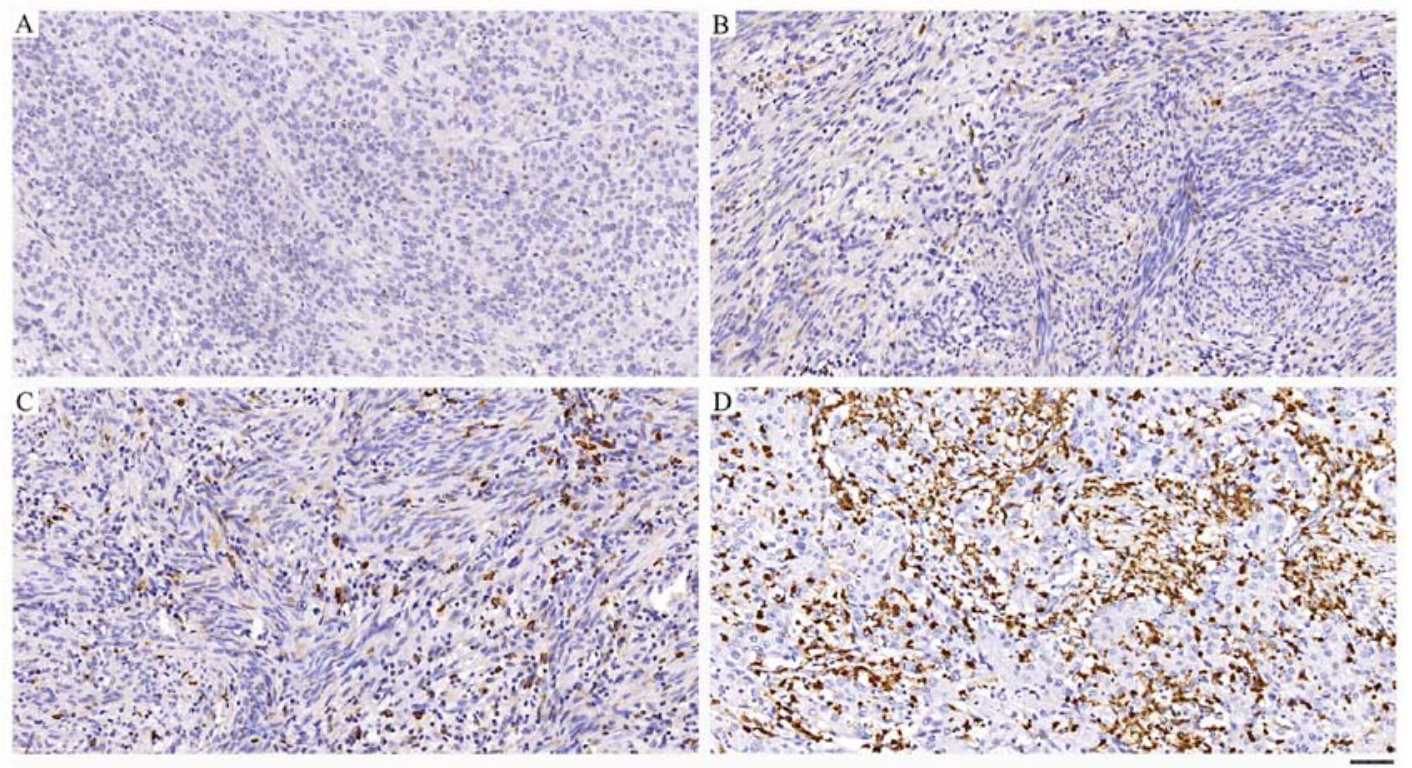

Figure 1. Immunohistochemical staining of HIF-1 $\alpha$ in locally advanced cervical cancer. Brown staining represents HIF-1 $\alpha$ expression, while blue staining represents the nuclei. (A) Negative control; (B) weighted score 1; (C) weighted score 4; (D) weighted score 9 (scale bar, $50 \mu$ m; magnification, x400). HIF-1 $\alpha$ was expressed in the nuclei and cytoplasm of tumor cells. HIF-1 $\alpha$, hypoxia-inducible factor-1 $\alpha$.

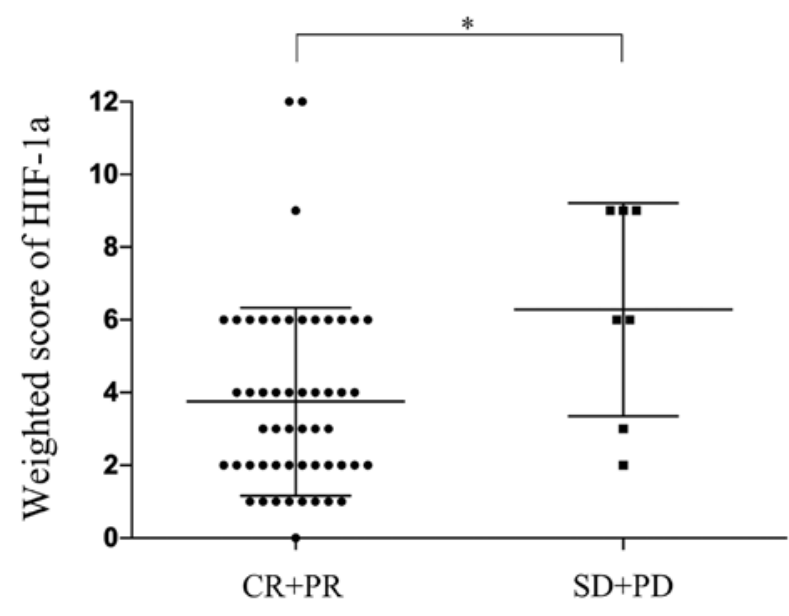

Figure 2. Weighted score for HIF-1 $\alpha$ expression in tumor samples from patients with locally advanced cervical cancer. HIF- $1 \alpha$ expression was significantly higher in the $\mathrm{SD}+\mathrm{PD}$ compared group with the $\mathrm{CR}+\mathrm{PR}$ group. ${ }^{*} \mathrm{P}=0.029$ (Mann-Whitney U test). CR + PR, complete remission + partial remission; $\mathrm{SD}+\mathrm{PD}$, stable disease + progressive disease; HIF-1 $\alpha$, hypoxia-inducible factor-1 $\alpha$.

poor control of micrometastasis, and the high incidence of permanent local toxicity due to radiation, mainly in young and sexually active women, have brought about the development of different therapeutic approaches such as NACT followed by radical surgery (32).

In clinical practice, only some patients with LACC benefit from chemotherapy treatment followed by radical surgery. Identifying patients who will be responsive to chemotherapy could provide them with proper treatment, which has important implications in personalized treatment and outcomes, while identifying non-responders may reduce the possibility of these patients receiving unsuccessful treatment and thereby enable them to receive more effective treatments as soon as the disease is diagnosed. Therefore, prognostic factors identifying

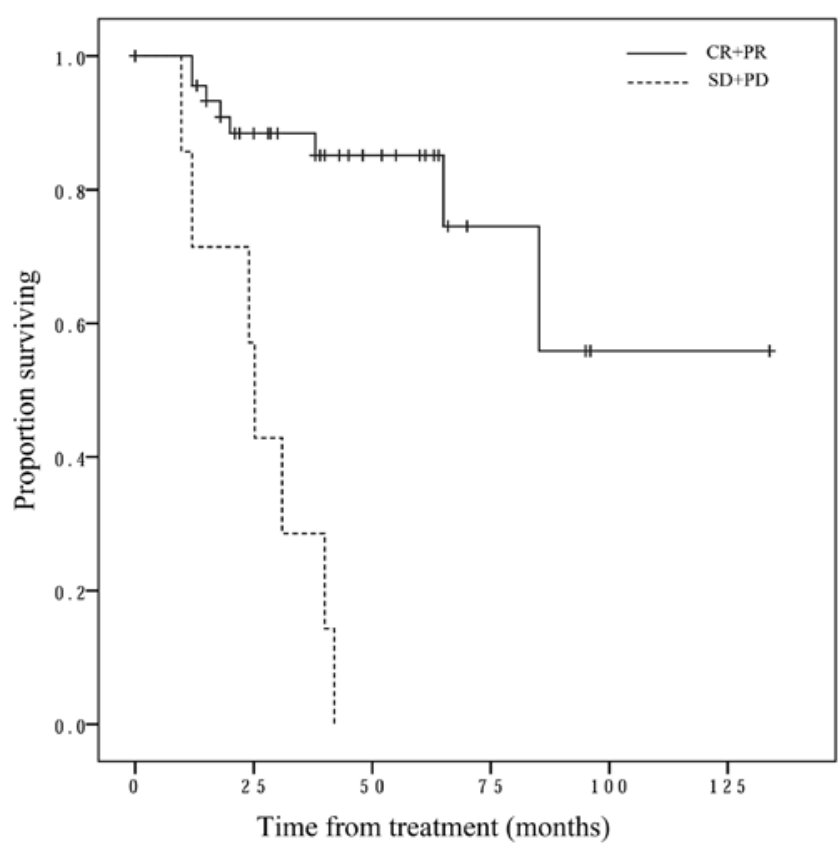

Figure 3. Overall survival rate in the $\mathrm{CR}+\mathrm{PR}(\mathrm{n}=52)$ and $\mathrm{SD}+\mathrm{PD}(\mathrm{n}=7)$ groups. Solid line, $\mathrm{CR}+\mathrm{PR}$; dashed line, $\mathrm{SD}+\mathrm{PD}$. CR + PR group exhibited significantly longer overall survival time compared with the SD + PD group $(\mathrm{P}<0.001$, Kaplan-Meier and log-rank tests). CR + PR, complete remission + partial remission; $\mathrm{SD}+\mathrm{PD}$, stable disease + progressive disease.

the efficiency of NACT will play a critical role in trials of NACT in these patients.

The main objective of NACT is to reduce tumor volume, reduce the clinical stages of the patients, decrease lymph node metastasis, increase the chance to achieve radical hysterectomy, preserve ovarian function and reduce the dose of postoperative radiation therapy, so as to improve the quality of life of patients, especially in sexually active woman (33-36). However, there are limitations to this strategy; if the NACT 
Table III. Characteristics of patients in the low and high HIF-1 $\alpha$ expression groups.

\begin{tabular}{|c|c|c|c|}
\hline Characteristics & Low HIF- $1 \alpha$ expression $\left(\leq 4^{a}\right)$ & High HIF- $1 \alpha$ expression $\left(\geq 6^{a}\right)$ & P-value \\
\hline Number of patients & 39 & 20 & \\
\hline Age, years (range) & $47(28-62)$ & $48(34-62)$ & $0.923^{\mathrm{b}}$ \\
\hline \multicolumn{4}{|l|}{ Clinical stage } \\
\hline Stage IIB & 36 & 20 & $0.544^{\mathrm{c}}$ \\
\hline Stage IIIA & 0 & 0 & \\
\hline Stage IIIB & 3 & 0 & \\
\hline \multicolumn{4}{|l|}{ Grade } \\
\hline G1 & 11 & 1 & $0.079^{c}$ \\
\hline $\mathrm{G} 2 / \mathrm{G} 3$ & 28 & 19 & \\
\hline \multicolumn{4}{|l|}{ Histology } \\
\hline $\mathrm{SCC}$ & 35 & 18 & $0.975^{\mathrm{c}}$ \\
\hline A & 4 & 2 & \\
\hline AS & 0 & 0 & \\
\hline Others & 0 & 0 & \\
\hline \multicolumn{4}{|l|}{ Size of tumor } \\
\hline$\leq 4 \mathrm{~cm}$ & 5 & 5 & $0.416^{\mathrm{c}}$ \\
\hline$>4 \mathrm{~cm}$ & 34 & 15 & \\
\hline \multicolumn{4}{|l|}{ Main therapy } \\
\hline $\mathrm{NACT}+\mathrm{S}+\mathrm{R}$ & 29 & 11 & $0.132^{\mathrm{c}}$ \\
\hline $\mathrm{NACT}+\mathrm{R}$ & 10 & 9 & \\
\hline
\end{tabular}

${ }^{a}$ Weighted score; ${ }^{b}$ independent two-sample t-test; ${ }^{c} \chi^{2}$ test. HIF- $1 \alpha$, hypoxia-inducible factor- $1 \alpha$; NACT $+\mathrm{S}+\mathrm{R}$, neoadjuvant chemotherapy + surgery + radiotherapy; NACT + R, neoadjuvant chemotherapy + radiotherapy; FIGO, International Federation of Gynecology and Obstetrics; SCC, squamous cell carcinoma; A, adenocarcinoma; AS, adenosquamous carcinoma.

Table IV. Number of patients with low and high HIF-1 $\alpha$ expression in the $\mathrm{CR}+\mathrm{PR}$ and $\mathrm{SD}+\mathrm{PD}$ groups.

\begin{tabular}{lccc}
\hline Expression & $\mathrm{CR}+\mathrm{PR}, \mathrm{n}(\%)$ & $\mathrm{SD}+\mathrm{PD}, \mathrm{n}(\%)$ & P-value \\
\hline Low, $\leq 4$ & $37(94.87)$ & $2(5.13)$ & $0.025^{\mathrm{a}}$ \\
High, $\geq 6$ & $15(75)$ & $5(25)$ & \\
\hline
\end{tabular}

${ }^{a} \chi^{2}$ test. HIF- $1 \alpha$, hypoxia-inducible factor- $1 \alpha$; CR + PR, complete remission + partial remission; $\mathrm{SD}+\mathrm{PD}$, stable disease + progressive disease.

is not administered efficiently, there will be a time delay and chemotherapy-induced resistance to radiotherapy, which would result in a worse prognosis $(8,9,37,38)$. Hence, it is crucial to identify factors that could predict the efficacy of NACT in patients with LACC.

Hypoxia, a decrease in oxygen concentration in the tissue microenvironment, affects physiological development and tumorigenesis (39). A key mediator of the response to hypoxia is HIF-1 $\alpha$ (40). HIF-1 $\alpha$ is inactive and remains at a low concentration in normoxia. In hypoxia, however, HIF-1 $\alpha$ is stabilized and activated. In gynecological cancers, HIF-1 $\alpha$ is an important factor in carcinogenesis, and high levels of HIF-1 $\alpha$ expression seem associated with shorter progression-free survival and
Table V. Cox regression multivariate analysis with overall survival as end point $(n=40)$.

\begin{tabular}{lccc}
\hline Risk factor & HR & 95\% CI for HR & P-value \\
\hline Lymph node metastasis & 6.909 & $1.356-35.216$ & 0.020 \\
High HIF-1 $\alpha$ expression & 6.354 & $1.262-31.995$ & 0.025 \\
Response to NACT & 0.246 & $0.026-2.541$ & 0.258 \\
\hline
\end{tabular}

HIF-1 $\alpha$, hypoxia-inducible factor- $1 \alpha$; NACT, neoadjuvant chemotherapy; HR, hazard ratio; CI, confidence interval.

overall survival (41). Significantly higher levels of HIF-1 $\alpha$ transcript and protein were detected in tumor tissue compared with normal tissue in cervical cancer (42). However, to the best of our knowledge, there is no study on the effect of HIF-1 $\alpha$ protein expression on chemoresistance of cervical cancer.

This retrospective study firstly reported that HIF-1 $\alpha$ protein expression may be able to distinguish patients with LACC at FIGO stage IIB and IIIB who are relatively chemoresistant. Also, it may be a good prognostic indicator over the current standards with clinical stage, since patients with the same clinical stage often have different prognosis (some patients are cured, while others suffer recurrence). The present results demonstrated that high levels of HIF-1 $\alpha$ expression were 


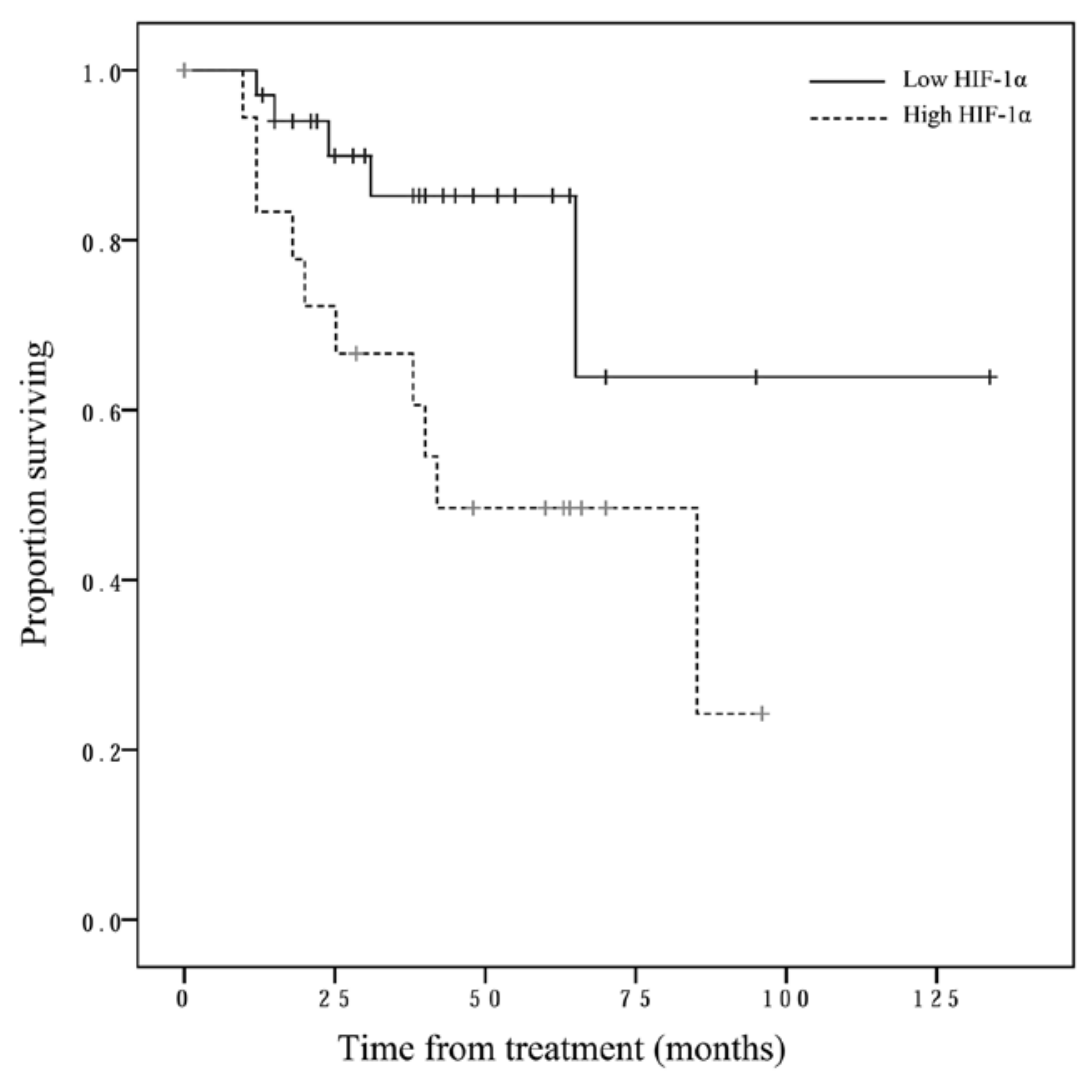

Figure 4. Overall survival rate in the low $(\mathrm{n}=39)$ and high HIF-1 $\alpha$ expression $(\mathrm{n}=20)$ groups. Solid line, low level of HIF-1 $\alpha$ expression; dashed line, high level of HIF-1 $\alpha$ expression. The low HIF-1 $\alpha$ group exhibited significantly longer overall survival time compared with the high HIF-1 $\alpha$ group (P=0.017, Kaplan-Meier and $\log$-rank tests). HIF-1 $\alpha$, hypoxia-inducible factor- $1 \alpha$.

associated with resistance to cisplatin-based chemotherapy and may be a prognostic predictor of the efficiency of NACT in patients with LACC at FIGO stage IIB and IIIB. In addition, survival analysis revealed that prognosis was worse when NACT was inefficient, which is in accordance with previous findings (7). Cox hazard analysis using post-surgery specimens indicated that lymph node metastasis and high levels of HIF-1 $\alpha$ expression were independent prognostic factors. However, NACT response was not an independent prognostic factor $(\mathrm{P}=0.258)$, which may due to the insufficient number of specimens and SD + PD patients. Further study is required to conduct experiments to detect HIF-1 $\alpha$ with multiple approaches such as quantitative PCR and western blotting to confirm these findings. The downstream molecules of HIF-1 $\alpha$, such as vascular endothelial growth factor (VEGF) and erythropoietin (EPO), should also be detected, as under a hypoxic environment, with increased levels of HIF-1 $\alpha$, VEGF and EPO promote angiogenesis and erythropoiesis, which will alter the hypoxic condition (43).

In conclusion, these results indicated that inefficient NACT for LACC leads to a worse prognosis. Therefore, factors that predict the efficiency of NACT will play an important role in trials of NACT for cancer. To the best of our knowledge, the present study is the first to show that the level of HIF-1 $\alpha$ expression may be a strong predictor of the efficiency of NACT and a prognostic factor in patients with stage IIB and IIIB cervical cancer. Future studies with larger patient numbers and different FIGO stage are required to validate and further elucidate this finding.

\section{Acknowledgements}

Not applicable.

\section{Funding}

The present study was supported by the National Natural Science Foundation of China (grant no. 81502265), Innovation Team Program of Science and Technology Department of Hubei Province (grant no. 2017CKC891), and General Program of Health and Safety Commission of Hubei Province (grant no. WJ2019M228).

\section{Availability of data and materials}

The datasets used and/or analyzed during the current study are available from the corresponding author upon reasonable request.

\section{Authors' contributions}

BY, XFW and JBH conceived and designed the study. BY, XFW, QFM, WFT, HNC, YLL, ZGZ, FXZ, YJX and $\mathrm{HG}$ performed the experiments and collected the data. BY, XFW, JBH, XD, MX and YLG analyzed and interpreted the data. BY and XFW drafted the initial manuscript and revised the important intellectual content. All authors read and approved the final version of the manuscript. 


\section{Ethics approval and consent to participate}

The research involving human samples was approved by the Ethics Committee of Maternal and Child Health Hospital of Hubei Province. All experiments were conducted according to relevant national and international guidelines. Informed consent was obtained from all participants included in the study.

\section{Patient consent for publication}

Informed consent was obtained from all participants included in the study.

\section{Competing interests}

The authors declare that they have no competing interests.

\section{References}

1. Gupta S, Maheshwari A, Parab P, Mahantshetty U, Hawaldar R, Sastri Chopra S, Kerkar R, Engineer R, Tongaonkar H, Ghosh J, et al: Neoadjuvant chemotherapy followed by radical surgery versus concomitant chemotherapy and radiotherapy in patients with stage IB2, IIA, or IIB squamous cervical cancer: A randomized controlled trial. J Clin Oncol 36: 1548-1555, 2018.

2. Morris M, Eifel PJ, Lu J, Grigsby PW, Levenback C, Stevens RE, Rotman M, Gershenson DM and Mutch DG: Pelvic radiation with concurrent chemotherapy compared with pelvic and para-aortic radiation for high-risk cervical cancer. N Engl J Med 340 : 1137-1143, 1999.

3. Keys HM, Bundy BN, Stehman FB, Muderspach LI, Chafe WE, Suggs CL III, Walker JL and Gersell D: Cisplatin, radiation, and adjuvant hysterectomy compared with radiation and adjuvant hysterectomy for bulky stage IB cervical carcinoma. N Engl J Med 340: 1154-1161, 1999.

4. Rose PG, Bundy BN, Watkins EB, Thigpen JT, Deppe G, Maiman MA, Clarke-Pearson DL and Insalaco S: Concurrent cisplatin-based radiotherapy and chemotherapy for locally advanced cervical cancer. N Engl J Med 340: 1144-1153, 1999.

5. Ishiko O, Sumi T, Yasui T, Matsumoto Y, Kawamura N, Ogita S, Kamino T, Nakamura K and Yamada R: Balloon-occluded arterial infusion chemotherapy, simple total hysterectomy, and radiotherapy as a useful combination-therapy for advanced cancer of the uterine cervix. Oncol Rep 7: 141-144, 2000.

6. Kawaguchi R, Nakamura H, Morioka S, Ito H, Tanase Y, Haruta S, Kanayama S, Yosida S, Furukawa N, Oi H and Kobayashi H: Comparison of neoadjuvant intraarterial chemotherapy versus concurrent chemoradiotherapy in patients with stage IIIB uterine cervical cancer. World J Oncol 4: 221-229, 2013.

7. Chen $\mathrm{H}$, Liang $\mathrm{C}$, Zhang L, Huang $\mathrm{S}$ and $\mathrm{Wu} \mathrm{X}$ : Clinical efficacy of modified preoperative neoadjuvant chemotherapy in the treatment of locally advanced (stage IB2 to IIB) cervical cancer: Randomized study. Gynecol Oncol 110: 308-315, 2008.

8. Souhami L, Gil RA, Allan SE, Canary PC, Araújo CM, Pinto LH and Silveira TR: A randomized trial of chemotherapy followed by pelvic radiation therapy in stage IIIB carcinoma of the cervix. J Clin Oncol 9: 970-977, 1991.

9. Tattersall MH, Lorvidhaya V, Vootiprux V, Cheirsilpa A, Wong F, Azhar T, Lee HP, Kang SB, Manalo A, Yen MS, et al: Randomized trial of epirubicin and cisplatin chemotherapy followed by pelvic radiation in locally advanced cervical cancer Cervical cancer study group of the Asian Oceanian clinical oncology association. J Clin Oncol 13: 444-451, 1995.

10. de la Torre M: Neoadjuvant chemotherapy in woman with early or locally advanced cervical cancer. Rep Pract Oncol Radiother 23: 528-532, 2018.

11. Yamauchi M, Fukuda T, Wada T, Kawanishi M, Imai K, Tasaka R, Yasui T and Sumi T: Expression of epidermal growth factor-like domain 7 may be a predictive marker of the effect of neoadjuvant chemotherapy for locally advanced uterine cervical cancer. Oncol Lett 12: 5183-5189, 2016.
12. Imai K, Fukuda T, Wada T, Kawanishi M, Tasaka R, Yasui T and Sumi T: UCP2 expression may represent a predictive marker of neoadjuvant chemotherapy effectiveness for locally advanced uterine cervical cancer. Oncol Lett 14: 951-957, 2017.

13. Okamoto E, Sumi T, Misugi F, Nobeyama H, Hattori K, Yoshida H, Matsumoto Y, Yasui T, Honda K and Ishiko O: Expression of apoptosis-related proteins in advanced uterine cervical cancer after balloon-occluded arterial infusion chemotherapy as an indicator of the efficiency of this therapy. Int J Mol Med 15: 41-47, 2005.

14. Nobeyama H, Sumi T, Misugi F, Okamoto E, Hattori K, Matsumoto $\mathrm{Y}$, Yasui $\mathrm{T}$, Honda $\mathrm{K}$, Iwai $\mathrm{K}$ and Ishiko $\mathrm{O}$ : Association of HPV infection with prognosis after neoadjuvant chemotherapy in advanced uterine cervical cancer. Int J Mol Med 14: 101-105, 2004.

15. Benedetti Panici P, Bellati F, Manci N, Pernice M, Plotti F, Di Donato V, Calcagno M, Zullo MA, Muzii L and Angioli R: Neoadjuvant chemotherapy followed by radical surgery in patients affected by FIGO stage IVA cervical cancer. Ann Surg Oncol 14: 2643-2648, 2007.

16. Karakashev SV and Reginato MJ: Progress toward overcoming hypoxia-induced resistance to solid tumor therapy. Cancer Manag Res 7: 253-264, 2015. Graham K and Unger E: Overcoming tumor hypoxia as a barrier to radiotherapy, chemotherapy and immunotherapy in cancer treatment. Int $\mathbf{J}$ Nanomedicine 13: 6049-6058, 2018.

17. Balamurugan K: HIF-1 at the crossroads of hypoxia, inflammation, and cancer. Int J Cancer 138: 1058-1066, 2016.

18. Jaakkola P, Mole DR, Tian YM, Wilson MI, Gielbert J, Gaskell SJ, von Kriegsheim A, Hebestreit HF, Mukherji M, Schofield CJ, et al: Targeting of HIF-alpha to the von Hippel-Lindau ubiquitylation complex by O2-regulated prolyl hydroxylation. Science 292: 468-472, 2001.

19. Lin SC, Chien CW, Lee JC, Yeh YC, Hsu KF, Lai YY, Lin SC and Tsai SJ: Suppression of dual-specificity phosphatase-2 by hypoxia increases chemoresistance and malignancy in human cancer cells. J Clin Invest 121: 1905-1916, 2011.

20. Chen Q, Tian WJ, Huang ML, Liu CH, Yao TT and Guan MM: Association between HIF-1 alpha gene polymorphisms and response in patients undergoing neoadjuvant chemotherapy for locally advanced cervical cancer. Med Sci Monit 22: 3140-3146, 2016.

21. Semenza GL: Defining the role of hypoxia-inducible factor 1 in cancer biology and therapeutics. Oncogene 29: 625-634, 2010.

22. Brown JM and Wilson WR: Exploiting tumour hypoxia in cancer treatment. Nat Rev Cancer 4: 437-447, 2004.

23. Iwasaki K, Yabushita H, Ueno $\mathrm{T}$ and Wakatsuki A: Role of hypoxia-inducible factor- $1 \alpha$, carbonic anhydrase-IX, glucose transporter-1 and vascular endothelial growth factor associated with lymph node metastasis and recurrence in patients with locally advanced cervical cancer. Oncol Lett 10: 1970-1978, 2015.

24. Kim BW, Cho H, Chung JY, Conway C, Ylaya K, Kim JH and Hewitt SM: Prognostic assessment of hypoxia and metabolic markers in cervical cancer using automated digital image analysis of immunohistochemistry. J Transl Med 11: 185, 2013.

25. Huang M, Chen Q, Xiao J, Yao T, Bian L, Liu C and Lin Z: Overexpression of hypoxia-inducible factor- $1 \alpha$ is a predictor of poor prognosis in cervical cancer: A clinicopathologic study and a meta-analysis. Int J Gynecol Cancer 24: 1054-1064, 2014.

26. Matsui Y, Kanoh H, Okudaira Y, Hashimoto T and Nakamura H: Superselective transcatheter-arterial-chemo-embolization in uterine cervical cancer. Gan To Kagaku Ryoho 16: 2801-2804, 1989 (In Japanese).

27. Tsuji K, Yamada R, Kawabata M, Mitsuzane K, Sato M, Iwahashi M, Kitayama S and Nakano R: Effect of balloon occluded arterial infusion of anticancer drugs on the prognosis of cervical cancer treated with radiation therapy. Int J Radiat Oncol Biol Phys 32: 1337-1345, 1995.

28. World Health Handbook for reporting results of cancer treatment. World Health Organization, Geneva, Offset Publication, No. 48, 1989.

29. Yan B, Wei JJ, Yuan Y, Sun R, Li D, Luo J, Liao SJ, Zhou YH, Shu Y, Wang Q, et al: IL-6 cooperates with G-CSF to induce protumor function of neutrophils in bone marrow by enhancing STAT3 activation. J Immunol 190: 5882-5893, 2013.

30. Sinicrope FA, Ruan SB, Cleary KR, Stephens LC, Lee JJ and Levin B: bcl-2 and p53 oncoprotein expression during colorectal tumorigenesis. Cancer Res 55: 237-241, 1995. 
31. National Comprehensive Cancer Network: NCCN Clinical Practice Guidelines in Oncology-Cervical Cancer-Version II, 2013.

32. Angioli R, Luvero D, Aloisi A, Capriglione S, Gennari P, Linciano F, Li Destri M, Scaletta G, Montera R and Plotti F: Adjuvant chemotherapy after primary treatments for cervical cancer: A critical point of view and review of the literature. Expert Rev Anticancer Ther 14: 431-439, 2014

33. Wada T, Fukuda T, Shimomura M, Inoue Y, Kawanishi M, Tasaka R, Yasui T, Ikeda $\mathrm{K}$ and Sumi T: XPA expression is a predictive marker of the effectiveness of neoadjuvant chemotherapy for locally advanced uterine cervical cancer. Oncol Lett 15: 3766-3771, 2018.

34. Ditto A, Martinelli F, Borreani C, Kusamura S, Hanozet F, Brunelli C, Rossi G, Solima E, Fontanelli R, Zanaboni F, et al: Quality of life and sexual, bladder, and intestinal dysfunctions after class III nerve-sparing and class II radical hysterectomies: A questionnaire-based study. Int J Gynecol Cancer 19: 953-957, 2009.

35. Robova H, Halaska M, Pluta M, Skapa P, Strnad P, Lisy J and Rob L: The role of neoadjuvant chemotherapy and surgery in cervical cancer. Int J Gynecol Cancer 20 (11 Suppl 2): S42-S46, 2010.

36. Iwata T, Miyauchi A, Suga Y, Nishio H, Nakamura M, Ohno A, Hirao N, Morisada T, Tanaka K, Ueyama H, et al: Neoadjuvant chemotherapy for locally advanced cervical cancer. Chin J Cancer Res 28: 235-240, 2016.

37. Landoni F, Sartori E, Maggino T, Zola P, Zanagnolo V, Cosio S, Ferrari F, Piovano E and Gadducci A: Is there a role for postoperative treatment in patients with stage Ib2-IIb cervical cancer treated with neo-adjuvant chemotherapy and radical surgery? An Italian multicenter retrospective study. Gynecol Oncol 132: 611-617, 2014.
38. Minig L, Colombo N, Zanagnolo V, Landoni F, Bocciolone L, Cárdenas-Rebollo JM, Iodice S and Maggioni A: Platinum-based neoadjuvant chemotherapy followed by radical surgery for cervical carcinoma international federation of gynecology and obstetrics stage IB2-IIB. Int J Gynecol Cancer 23: 1647-1654, 2013.

39. Semenza GL: HIF-1: Upstream and downstream of cancer metabolism. Curr Opin Genet Dev 20: 51-56, 2010.

40. Wang GL, Jiang BH, Rue EA and Semenza GL: Hypoxia-inducible factor 1 is a basic-helix-loop-helix-PAS heterodimer regulated by cellular O2 tension. Proc Natl Acad Sci USA 92: 5510-5514, 1995.

41. Seeber LM, Horrée N, Vooijs MA, Heintz AP, van der Wall E, Verheijen RH and van Diest PJ: The role of hypoxia inducible factor-1alpha in gynecological cancer. Crit Rev Oncol Hematol 78: 173-184, 2011.

42. Łuczak MW, Roszak A, Pawlik P, Kędzia H, Lianeri M and Jagodziński PP: Increased expression of HIF-1A and its implication in the hypoxia pathway in primary advanced cervical carcinoma. Oncol Rep 26: 1259-1264, 2011.

43. Masoud GN and Li W: HIF-1 $\alpha$ pathway: Role, regulation and intervention for cancer therapy. Acta Pharm Sin B 5: 378-389, 2015.

This work is licensed under a Creative Commons Attribution-NonCommercial-NoDerivatives 4.0 International (CC BY-NC-ND 4.0) License. 